\title{
Femtosecond electron transfer: Experiment and theory
}

\author{
Alan E. Johnson, Keisuke Tominaga, Gilbert C. Walker, Wlodzimierz Jarzeba, \\ and Paul F. Barbara
}

Department of Chemistry, University of Minnesota, Minneapolis, MN 55455 U.S.A.

\begin{abstract}
The electron transfer kinetics of 4-(9-anthryl)-N,N'-dimethylaniline (ADMA) and 9,9'-bianthryl (BA) were measured. The dynamics of BA and ADMA are both well described with a semi-empirical model for electron transfer. Within this model, the BA electron transfer dynamics can be described as occurring adiabatically in $S_{1}$. However, in ADMA, part of the electron transfer occurs nonadiabatically from $S_{2}$ to $S_{1}$ and part of the electron transfer occurs adiabatically within $S_{1}$. To describe the combined adiabatic and nonadiabatic dynamics in ADMA, we introduce a generalized survival probability related to the fractional charge remaining on the donor.In addition, the dipolar solvation dynamics of an anionic probe (conjugate base of coumarin 343) molecule were measured in methanol and found to be similar to previously reported solvation dynamics of a neutral probe. Also, no evidence of ion pairing contributions to the solvation dynamics were observed.
\end{abstract}

\section{INTRODUCTION}

Photophysical studies on organic aromatic electron donor/acceptor compounds are leading to new information on several issues of contemporary interest, including the role of solvation dynamics in electron transfer reactions (ref. 1). This area is focused on the limitations of the traditional models for electron transfer, such as Marcus-Hush theory, which are based on the quasi-equilibrium approximation for the distribution along the solvent coordinate, and, consequently rule out a kinetic role of solvation dynamics. Experiment, on the other hand, has demonstrated that dynamic solvent effects can be significant in many cases, especially for electron transfer reactions with small intrinsic activation barriers, i.e. $\Delta \mathrm{G} \ddagger<<\mathrm{k}_{\mathrm{B}} \mathrm{T}$.

This paper describes the experimental solvation dynamics of an ionic probe, the conjugate base of coumarin 343 (C343) in methanol, and the experimental electron transfer dynamics of 9,9'-bianthryl (BA) (refs. 2-3) and 4-(9-anthryl)-N, $\mathrm{N}^{\prime}$-dimethylaniline (ADMA) (refs. 4-6) in polar organic solvents. The molecules are depicted in Figure 1. The technique used for these measurements was femtosecond fluorescence upconversion. The electron transfer dynamics are compared to a phenomenological model for electron transfer which includes the response of solvation dynamics (refs. $3,5-6$ ).<smiles>O=C(O)c1cc2cc3c4c(c2[se]c1=O)CCCN4CCC3</smiles>

C343<smiles>c1ccc2c(-c3c4ccccc4cc4ccccc34)c3ccccc3cc2c1</smiles>

BA<smiles>CN(C)c1ccc(-c2c3ccccc3cc3ccccc23)cc1</smiles>

ADMA

Figure 1. Structures of C $\$ 43, B A$, and $A D M A$. 


\section{II. $\mathrm{C343}$}

We have studied the solvation dynamics of the conjugate base of C343 in methanol and find the dynamics to be similar to those of the neutral probe C152 (ref. . In the absence of base, C343 exists as a neutral molecule with an absorption maximum of $446 \mathrm{~nm}$ in methanol. With the addition of $\mathrm{NaOCH}_{3}$, the absorption spectrum shifts to the blue due to deprotonation of the carboxylic acid group of C343. Similarly, the fluorescence spectrum also shifts to the blue with the addition of base.

Time resolved fluorescence measurements (resolution $\simeq 150 \mathrm{fs}$ ) were performed at 9 wavelengths with an excess of base to insure complete deprotonation. From these fluorescence transients, we calculated the fluorescence spectrum as a function of time, spectral reconstruction. From the spectral reconstruction, we obtained the empirical solvation function, $C(t)$, defined as

$$
\mathrm{C}(\mathrm{t})=\frac{\nu(\mathrm{t})-\nu(\infty)}{\nu(0)-\nu(\infty)}
$$

Here, $\nu(t)$ represents the emission maximum at time $t$ after excitation. This $C(t)$ function can be used as input to calculations of barrierless electron transfer rates. In the present case, the $C(t)$ function is well fit by a biexponential function with time constants (and amplitudes) $1.0 \mathrm{ps}$ (21\%) and $10.3 \mathrm{ps}(79 \%)$, giving an average time of $8.3 \mathrm{ps}$. These times are similar to those previously measured for the neutral probe, $\mathrm{C} 152$, in methanol; the values are $1.2 \mathrm{ps}(40 \%)$ and $9.6 \mathrm{ps}(60 \%)$ for an average time of $6.1 \mathrm{ps}$ (ref. 7). Finally, we found no significant dependence of the solvation dynamics on the amount of excess base, indicating that ion-paring effects are not important in the solvation dynamics.

\section{BA}

The dynamics of the excited state electron transfer of electronically excited BA in polar solvents have been modeled using a semi-empirical theoretical model, due to Kang et al. (ref. 3), for the reaction coordinate energy profile and the dynamics along the reaction coordinate. The predictions of the model are in excellent agreement with the femtosecond fluorescence upconversion data on bianthryl. The model invokes an Onsager cavity / semi-empirical treatment for the solvent coordinate and uses an electronically adiabatic description of the mixing between reactant and product zero-order states, as shown in Figure 2. Motion along the solvent coordinate is modeled using a generalized Langevin equation treatment of the reaction coordinate dynamics. The friction kernel is determined using independent experimental results on solvation dynamics of coumarin probes, as described in section II. Finally, an empirical solvatochromic/vibronic description is used to predict fluorescence and absorption spectra. With a limited amount of parameterization the overall model is able to account in detail for many observables for BA, including the static absorption spectra, the solvent dependence of the static fluorescence spectra, and the time resolved fluorescence spectra. The model supports our previous proposal that the electron transfer kinetics of bianthryl is controlled by polar solvation dynamics.

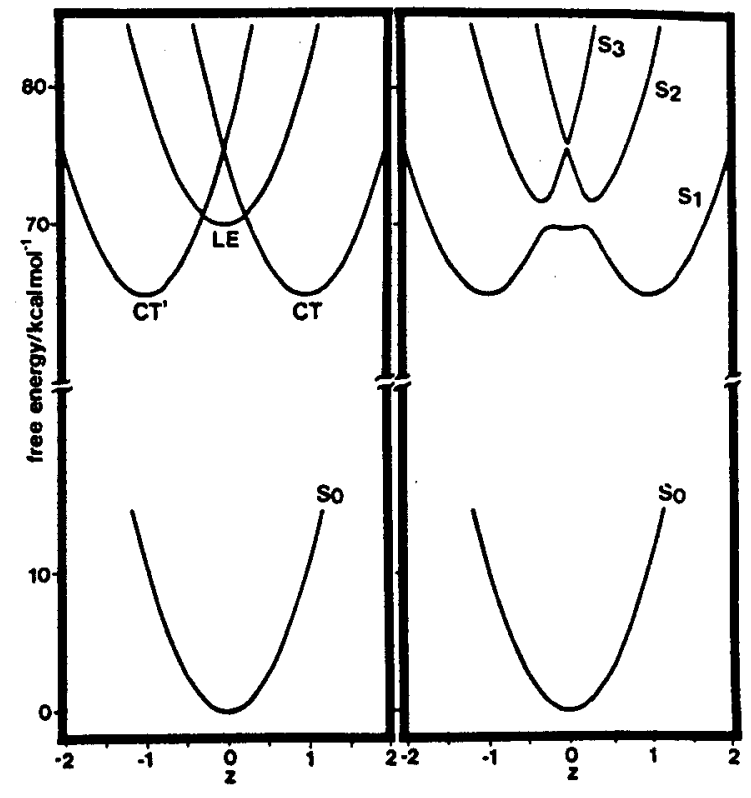

Figure 2. Theoretical estimates for the diabatic (left) and adiabatic free energies of $B A$ in propylene carbonate as a function of the solvent coordinate. 
Briefly, the model considers four diabatic states, the ground state $\left(S_{0}\right)$ the locally excited (LE) state, in which the excitation is localized on one anthracene, and two charge transfer states (CT and ('T') which correspond to the two possible directions of charge separation. The three excited states are mixed to form the adiabatic states, $S_{1}, S_{2}$, and $S_{3}$, as shown in Figure 2.

$$
\left|\Psi_{\mathrm{S}_{1}}>=\mathrm{c}_{1 \mathrm{LE}}\right| \Psi_{\mathrm{LE}}>+\mathrm{c}_{2} \mathrm{CT}\left|\Psi_{\mathrm{CT}}>+\mathrm{c}_{3} \mathrm{CT},\right| \Psi_{\mathrm{CT}^{\prime}}>
$$

The solvent reorganization energy, free energy difference, and mixing coefficients needed to describe the electronic states are obtained by fitting the absorption and fluorescence spectra. The absorption spectrum is fit to a convolution of the equilibrium ground state population distribution function and a vibronic shape function. The emission spectrum is fit to a convolution of the equilibrium $S_{1}$ population distribution and a vibronic shape function. In both cases, the experimentally observed absorption spectrum in hexane (reflected for fluorescence) as the vibronic shape function.

Population dynamics in $S_{1}$ were calculated in various solvents using the generalized Langevin equation with a friction kernel determined by the solvation correlation function, $C(t)$ and surface, $S_{1}$ for that solvent. Population distribution functions at specific times were convoluted with the vibronic shape function to yield the time dependent fluorescence spectrum for comparison with the femtosecond fluorescence upconversion data.

\section{ADMA}

We have extended the semi-empirical model of Kang et al. to describe the intramolecular excited state electron transfer in ADMA (refs. 5-6). The zero order and the adiabatic states are shown in Figure 3. In this case, only three diabatic states, $S_{0}, L E$, and CT, are required because the donor and acceptor have different identities. Predictions of the static absorption and emission spectra using these states are in good agreement with experiment, leading to the conclusion that the variation in the static absorption and emission spectra of ADMA, as a function of solvent, is primarily due to simple "solvent coordinate" effects, rather than large intramolecular structural changes, as in BA. In polar solvents, the excited state electron transfer $(\mathrm{LE} \rightarrow \mathrm{CT})$ of ADMA is in the Marcus inverted regime, see Figure 3. Experimentally, we observe two distinct electron transfer kinetic components, a faster ( $<150 \mathrm{fs})$ component which is not solvent controlled and probably involves intramolecular vibrations, and a slower solvent-controlled component with a limiting rate constant of $\approx 1 /\left\langle\tau_{\mathrm{s}}\right\rangle$, where $\left\langle\tau_{\mathrm{s}}\right\rangle$ is the average solvation time. Simulations employing the Kang et al. model are in semiquantitative agreement with all static and dynamics data on ADMA.
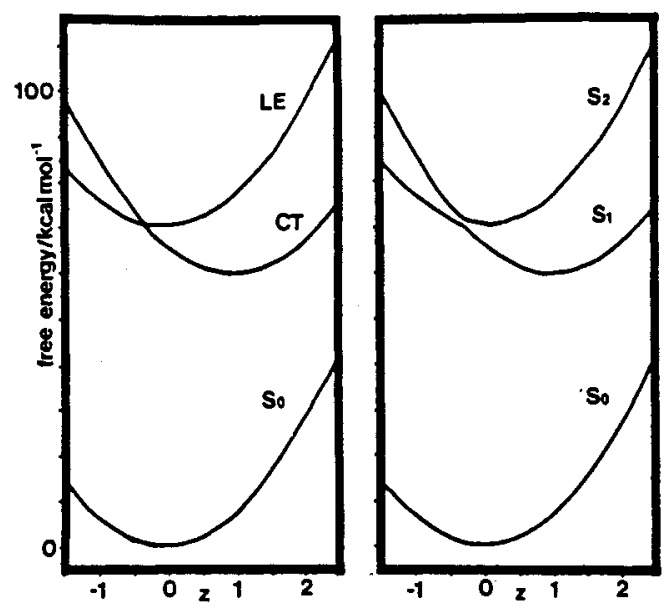

Figure 3. Theoretical estimates for the diabatic (left) and adiabatic (right) free energies of ADMA in $N, N^{\prime}$-dimethylformamide as a function of the solvent coordinate.

Static absorption and emission spectra were calculated using the adiabatic states, as in BA. Unlike $B A$, however, $S_{2}$ had to be included in the simulation of the absorption spectrum. This is because, unlike BA, the energy gap between $S_{1}$ and $S_{2}$ is small in the Franck-Condon region. The static emission spectrum was calculated using the equilibrium population distribution in $S_{1}$. Population dynamics in $S_{1}$ were calculated by numerical integration of the generalized diffusion equation, again using the solvation correlation function, $C(t)$, as an input. Population relaxation from $S_{2}$ to $S_{1}$ was assumed to occur much faster than solvation dynamics and corresponds to the experimentally observed fast component to electron transfer. 
To treat both types of electron transfer in ADMA, we have generalized this phenomenological model of electron transfer to include simultaneously two types of electron transfer (ref. 6), namely adiabatic electron transfer in $S_{1}$ and nonadiabatic electron transfer $\left(S_{2}-S_{1}\right)$. A general definition of the electron transfer "reactant" survival probability can be given in terms of the surviving charge on the donor, $\mathrm{S}_{\mathrm{G}}(\mathrm{t})$. The generalized electron transfer rate coefficient, $\mathbf{k}_{\mathbf{E T}}(\mathrm{t})$, can be expressed as

$$
k_{E T}=-\frac{d}{d t} \ln \left(S_{G}(t)-S_{G}(w)\right)
$$

A relationship between the survival probability, $S_{G}(t)$, defined in this manner and the integrated emission intensity from $S_{1}$ and $S_{2}, A(t)$, can then be established. In the nonadiabatic limit, in which the coupling between donor and acceptor is weak, the simple limit that the survival probability is proportional to the integrated intensity results.

\section{CONCLUSIONS}

We have observed the solvation dynamics of an ionic probe in methanol and find them to be very similar to previously measured results with a neutral probe. In addition, we did not observe evidence for ion pairing dynamics at higher concentrations of base. We have also developed a semi-empirical method for predicting electron transfer dynamics using parameters determined from the static absorption and fluorescence spectra. For simple barrierless electron transfer, as in BA, an adiabatic treatment is sufficient. For inverted regime reactions, as in ADMA, a combination of adiabatic and nonadiabatic dynamics are important. To describe the dynamics in such a case, we have defined the electron transfer rate in terms of a generalized charge survival probability on the donor.

\section{Acknowledgements}

Support for this research by the NSF is gratefully acknowledged.

\section{REFERENCES}

1. For recent reviews on excited-state electron transfer, see: J.D. Simon, Acc. Chem. Res., 21, 128-34 (1988); P.F. Barbara and W. Jarzeba, Adv. Photochem., 15, 1-68 (1990); M. Maroncelli, J. MacInnis, and G.R. Fleming, Science, 243, 1674-81 (1989); E. Lippert, W. Rettig, V. Bonacic-Koutecky, F. Heisel, and J.A. Miehe, Adv. Chem. Phys., 68, 1-174 (1987).

2. N. Mataga, H. Yao, T. Okada, and W. Rettig, J. Phys. Chem., 93, 3383-6 (1989); T.J. Kang, M.A. Kahlow, D. Giser, S. Swallen, V. Nagarajan, W. Jarzeba, and P.F. Barbara, J. Phys. Chem., 92, 6800-7 (1988); M.A. Kahlow, T.J. Kang, and P.F. Barbara, J. Phys. Chem., 91, 6452-5 (1987); H. Lueck, M.W. Windsor, and W. Rettig, J. Phys. Chem., 94, 4550-9 (1990); D.B. Toublanc, R.W. Fessenden, and A. Hitachi, J. Phys. Chem., 93, 2893-6 (1989); W. Rettig, and M. Zander, Ber. Bunsenges. Physik. Chem., 87, 1143-9 (1983).

3. T.J. Kang, W. Jarzeba, P.F. Barbara, and T. Fonseca, Chem. Phys., 149, 81-95 (1990).

4. T. Okada, N. Mataga, W. Baumann, and A. Siemiarczuk, J. Phys. Chem., 91, 4490-5 (1987); N. Mataga, S. Nishikawa, T. Asahi, and T. Okada, J. Phys. Chem., 94, 1443-7 (1990); A. Siemiarczuk, Z.R. Grabowski, A. Krowczynski, M. Asher, and M. Ottolenghi, Chem. Phys. Lett., 51, 315-20 (1977); Z.R. Grabowski, K. Rotkiewicz, A. Siemiarczuk, D.J. Cowley, and W. Baumann, Nouv. J.' Chim., 3, 443-54 (1979); A.M. Rollinson and H.G. Drickamer, J. Chem. Phys. 73, 5981-96 (1980); V. Nagarajan, A.M. Brearley, T.J. Kang, and P.F. Barbara, J. Chem. Phys., 86, 3183-96 (1987); D. Huppert, and P.M. Rentzepis, J. Phys. Chem., 92, 5466-9 (1988).

5. K. Tominaga, G.C. Walker, W. Jarzeba, and P.F. Barbara, J. Phys. Chem., 95, 10475-85 (1991).

6. K. Tominaga, G.C. Walker, T.J. Kang, P.F. Barbara, and T. Fonseca, J. Phys. Chem., 10485-92 (1991).

7. M. Maroncelli, J. Mol. Liquids, in press. 